\title{
Nanochains Formation of Superparamagnetic Nanoparticles
}

\author{
G. Bertoni, ${ }^{*,+}$ B. Torre, ${ }^{\dagger}$ A. Falqui, ${ }^{\dagger}$ D. Fragouli, ${ }^{\neq}$A. Athanassiou, ${ }^{+,}{ }^{+}$and R. Cingolani ${ }^{\dagger}$ \\ ${ }^{\dagger}$ Italian Institute of Technology (IIT), via Morego 30, I-16163 Genoa, Italy \\ ${ }^{\ddagger}$ Center for Bio-Molecular Nanotechnologies (CBN) of IIT@UniLe, via Barsanti, I-73010 Lecce, Italy
}

ABSTRACT: We present simulations on the aggregation of nanometer sized polydispersed superparamagnetic particles under the application of an external magnetic field. We make use of a Monte Carlo method, using a cluster-moving approach, as previously used in literature for ferrofluids. van der Waals attraction and magnetic anisotropy are taken into account in the simulations. Chains elongated in the field direction are formed. The results are in good agreement with recent experimental results on nanochains made of iron oxide nanoparticles into polymer matrix, obtained with the application of a magnetic field during film deposition. The magnetization anisotropy of the nanocomposite film under dc magnetic field can be predicted within this simple model.

\section{INTRODUCTION}

Nanocomposite films consisting in colloidal nanoparticles inside polymers attract much interest in view of the possibility of realizing plastic films with tuned characteristics. ${ }^{1-3}$ For instance, composite materials made of magnetic particles dispersed in a nonmagnetic elastic matrix are nowadays studied for applications in mechanical vibration sensors. ${ }^{4}$ Recently, ${ }^{5,6}$ we have shown the formation of nanochains structures made of iron oxide $\left(\gamma-\mathrm{Fe}_{2} \mathrm{O}_{3}\right)$ $\left.\mathrm{Fe}_{3} \mathrm{O}_{4}\right)$ nanoparticles ${ }^{7}$ inside polymers films, obtained by applying a magnetic field $(0.160 \mathrm{~T})$ during film drying. The chains (or wires) are micrometers long (Figure 1), whereas maintaining the superparamagnetic character of the constituent particles. Moreover, we have found that films with these wires exhibit anisotropic behavior of magnetization under weak fields (Figure 8), as revealed by SQUID magnetic measurements, opening the possible application of these plastic films in magnetic sensors, or magnetic shields. Superparamagnetism ${ }^{8,9}$ is a particular behavior of small magnetic particles. Below a critical size, depending on both exchange and magnetocrystalline anisotropy constant of the material, Bloch walls formation is not energetically favored. At sufficient high temperature, the thermal energy can flip the particles magnetic moments. In this regime, the magnetic behavior of an ensemble of such particles mimics the Langevin paramagnetism of atoms, but with a moment of a particle up to $10^{5}$ times the atomic moment (i.e., superparamagnetism). In this manuscript, we describe the simulation procedure of chainlike structure formation of superparamagnetic nanoparticles. The aim is to predict within a simple model the aggregation of nanoparticles also in a polymer matrix and the behavior of the film under a magnetic field, comparing the results of simulations with the experiment.

\section{METHODS}

Experiment. The chemical synthesis of the $\gamma-\mathrm{Fe}_{2} \mathrm{O}_{3} / \mathrm{Fe}_{3} \mathrm{O}_{4}$ nanoparticles, and the experimental procedure for obtaining the

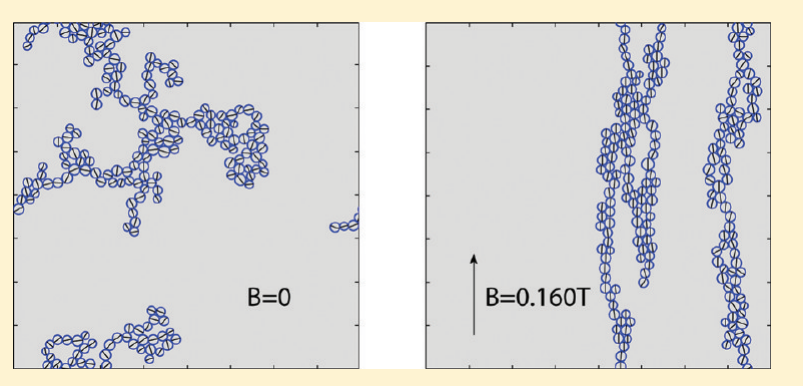

polymer films are described elsewhere, ${ }^{5-7}$ together with the structural and magnetic characterization of the constituent particles. Two sizes of particles are considered here, with nominal diameters of $\sim 11$ and $\sim 17 \mathrm{~nm}$. The TEM and STEM images of the pristine particles were acquired on a JEOL JEM-2200FS operating at $200 \mathrm{kV}$ and equipped with an energy filter (Omega type). Filtered elastic images (see for instance left panel of Figure 2) revealed the presence of the surfactant capping molecules surrounding the particles, mainly consisting of Oleic acid (OLAC). ${ }^{5}$ Two fundamental parameters needed in the simulation are the average diameter $d_{0}$, and the capping thickness $\delta$ (Figure 3). STEM images can be used to measure the particles core diameters, due to the high contrast of the magnetic core with respect to the capping layer. The average core diameter $d_{0}$ can be obtained using particle analysis procedures (as the one in Image $\mathrm{J}^{10}$ ). The average nearest-neighbor distance between particles can be statistically measured from the Fourier transform (FFT) of the STEM image, by assuming a negligible role of the adhesion with the carbon thin film support. An average effective capping thickness $\delta$ can then be determined using the potentials from Figure 2, by searching the value of $\delta$ that gives the minimum of the potential at the measured interparticle distance. The same procedure can be used on the thin slices from the obtained nanocomposite films, with the difficulty that the wires in TEM/ STEM images are $2 \mathrm{D}$ projection of a $3 \mathrm{D}$ object. The measured values for the diameters were $d_{0}=10.9 \mathrm{~nm}$ with a calculated capping thickness $\delta=0.85 \mathrm{~nm}$ (i.e., $\delta=0.08 d_{0}$ ) for the small particles, and $d_{0}=17.3 \mathrm{~nm}$ with $\delta=0.87 \mathrm{~nm}$ (i.e., $\delta=0.05 d_{0}$ ) for the bigger particles. The relative standard deviation in the diameter dispersion for both cases was found below 0.1 .

Calculation. Simulations were carried out using a Monte Carlo method, ${ }^{11}$ based on the Metropolis algorithm. ${ }^{12}$ The system

Received: November 25, 2010

Revised: January 27, 2011

Published: March 29, 2011 

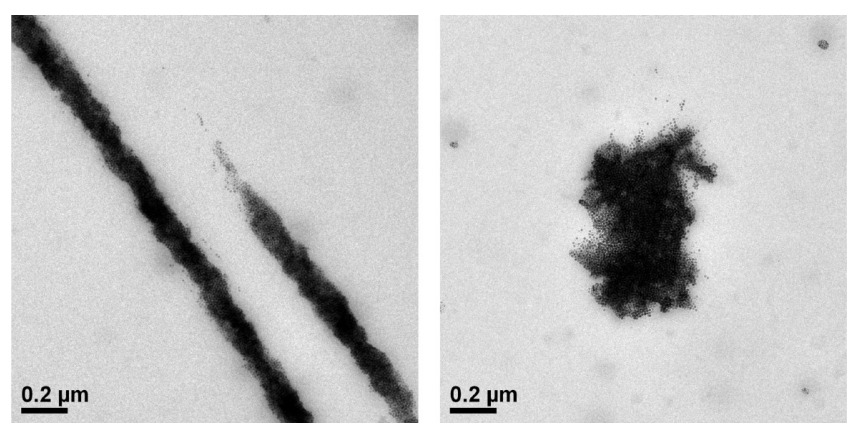

Figure 1. (left) TEM image of the obtained nanochains in PEMMA after application of a magnetic filed of $0.160 \mathrm{~T}$ during polymer drying, and (right) without the application of the magnetic field. ${ }^{5}$
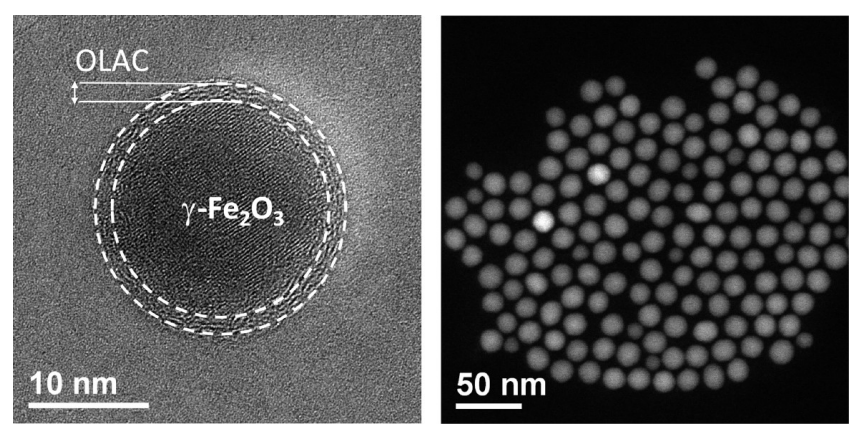

Figure 2. (left) Elastic filtered TEM image of a $\sim 17 \mathrm{~nm}$ particle after deposition on a carbon grid. Because of the enhanced contrast the capping layer (indicated by the white dashed lines) can be revealed. (right) STEM image of the particles, from which an average diameter of $17.3 \pm 1.8 \mathrm{~nm}$ and an average distance of $18.9 \mathrm{~nm}$ were deduced.

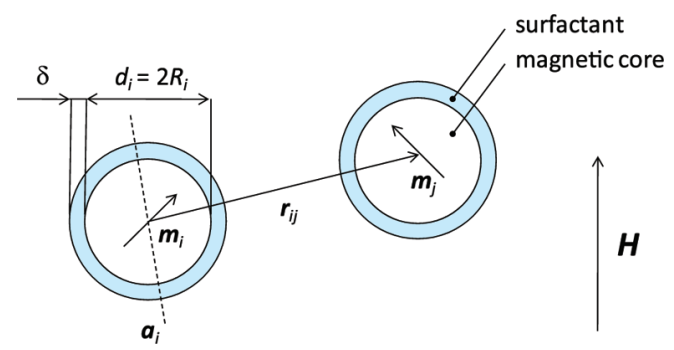

Figure 3. Scheme of the particle interaction used in the simulation.

consists of $N$ particles dispersed in a 2D box, with diameters $d_{i}$, normally distributed with average diameter $d_{0}$. The particles have a superparamagnetic core with magnetic moment $m_{i}$ and are covered by a steric layer of thickness $\delta$ (Figure 3 ). We adopted a cluster-moving algorithm of the type described by Satoh. ${ }^{13}$ Following a similar notation, ${ }^{14}$ we indicate the single particle term, due to the interaction with the external field, as $u_{i}^{(H)}$ (eq 1 in 14 modified here into eq 1) by including an effective uniaxial anisotropy (volume dependent) at first order, according to a Stoner-Wohlfart model. ${ }^{15}$ The single particle energy term is in this case changed in

$$
u_{i}^{(H)}=-k T \xi_{i} \mathbf{n}_{i} \cdot \mathbf{H} / H+K_{i}\left(1-\left(\mathbf{n}_{i} \cdot \mathbf{a}_{i}\right)^{2}\right)
$$

where $\mathbf{a}_{i}$ is the direction of easier magnetization, and the dimensionless parameter $\xi_{i}$ is given by

$$
\xi_{i}=\left(\frac{d_{i}}{d_{0}}\right)^{3} \xi_{0}
$$

with $\xi_{0}=\mu_{0} m_{0} H / k T$. Accordingly, the anisotropy barrier $K_{i}$ is given by

$$
K_{i}=\left(\frac{d_{i}}{d_{0}}\right)^{3} K_{0} V_{0}
$$

with $K_{0}$ an effective anisotropy constant. As can be seen from Figure 4, the anisotropy constant $K_{0}$ is slowly varying for diameters above $10 \mathrm{~nm}$ (as in the present cases), and therefore it is fixed to the value corresponding to the average volume $V_{0}$ (calculated from the average diameter $d_{0}$ ). The (dipolar) magnetic interaction between particles pairs is indicated as $u_{i j}^{(m)}$ (eq 2 in 14),

$$
u_{i j}^{(m)}=k T \lambda_{i j} \frac{d_{0}^{3}}{r_{i j}^{3}}\left\{\mathbf{n}_{i} \cdot \mathbf{n}_{j}-3\left(\mathbf{n}_{i} \cdot \mathbf{t}_{i j}\right)\left(\mathbf{n}_{j} \cdot \mathbf{t}_{i j}\right)\right\} \cdot
$$

The dimensionless parameter $\lambda_{i j}$ is given by

$$
\lambda_{i j}=\left(\frac{d_{i} d_{j}}{d_{0}^{2}}\right)^{3} \lambda_{0}
$$

where $\lambda_{0}=\mu_{0} m_{0}^{2} / 4 \pi d_{0}^{3} k T, \mathbf{n}_{i}=\mathbf{m}_{i} / m_{i}$, and $\mathbf{t}_{i j}=\mathbf{r}_{i j} / r_{i j}$, with $r_{i j}$ the distance between particles $i$ and $j$. The repulsion due to the surfactant layer is $\boldsymbol{u}_{i j}^{(v)}$ (from eq 5 in 14)

$$
\begin{aligned}
u_{i j}^{(v)}= & \frac{\lambda_{v i} k T}{2}\left\{2-\frac{2 C_{i}}{t_{\delta i}} \ln \left(\frac{t_{\delta i}+1}{C_{i}}\right)-2 \frac{C_{i}-1}{t_{\delta i}}\right\} \\
& +\frac{\lambda_{v j} k T}{2}\left\{2-\frac{2 C_{j}}{t_{\delta i}} \ln \left(\frac{t_{\delta i}+d_{j} / d_{i}}{C_{j}}\right)-2 \frac{C_{j}-d_{j} / d_{i}}{t_{\delta i}}\right\}, \\
C_{i}= & \frac{\left(1+t_{\delta i}\right)-\left(d_{j} / d_{i}+t_{\delta i}\right)^{2}+4 r_{i j}^{2} / d_{i}}{4 r_{i j} / d_{i}}, \\
C_{j}= & \frac{\left(d_{j} / d_{i}+t_{\delta i}\right)-\left(1+t_{\delta i}\right)^{2}+4 r_{i j}^{2} / d_{i}}{4 r_{i j} / d_{i}}
\end{aligned}
$$

in which $t_{\delta i}$ is the ratio of the thickess of the steric layer with respect to the radius of the particle $i$, that is $2 \delta / d_{i}$, and the dimensionless parameter $\lambda_{v i}$ is given by

$$
\lambda_{v i}=\left(\frac{d_{i}}{d_{0}}\right)^{2} \lambda_{v}
$$

with strength $\lambda_{v}=\pi d_{0}^{2} n_{\mathrm{s}} / 2$. In these expression $k$ is the Bolztmann's constant, $T$ the absolute temperature of the fluid, and $n_{\mathrm{s}}$ is the number of surfactant molecules per unit area on the particle surface. We included the attractive interaction, ${ }^{16}$ due to the van der Waals force between pairs $u_{i j}^{(\mathrm{vdW})}$, by generalizing eq 7 in 16 for a polydisperse system, in

$u_{i j}^{(v d W)}=-\frac{A}{6}\left\{\frac{2 R_{i} R_{j}}{r_{i j}^{2}-\left(R_{i}+R_{j}\right)^{2}}+\frac{2 R_{i} R_{j}}{r_{i j}^{2}-\left(R_{i}-R_{j}\right)^{2}}+\ln \left(\frac{r_{i j}^{2}-\left(R_{i}+R_{j}\right)^{2}}{r_{i j}^{2}-\left(R_{i}-R_{j}\right)^{2}}\right)\right\}$

where $A$ is the Hamaker's constant, and $R_{i}$ is the radius of particle $i$. The pair potentials are presented in Figure 5 for the 11 and $17 \mathrm{~nm}$ particles, respectively, for $T=300 \mathrm{~K}$, corresponding to the temperature of synthesis of the composites films. All simulations are done at the same temperature. In summary, ${ }^{13}$ at each iteration 
in the Monte Carlo algorithm, a trial movement on a random particle $i$ is tried, with a maximum displacement of $0.5 d_{i}$ for the particle, and a maximum change in orientation of $10^{\circ}$. The rotation is considered for both the anisotropy axis (accounting for the Brownian rotation of the particle), and for the magnetic moment (Neél rotation), independently. The total energy $U$ of the system is the calculated by summing the terms in eqs $1-8$, and the movement is accepted with probability $\exp (-\Delta U / k T) .{ }^{11}$ To speed up convergence, at some points (i.e., after a serie of single particle movements) the formation of clusters of particles is checked. A cluster is formed when the distance between particles is below a critical value. ${ }^{13}$ If a cluster is formed, it is allowed to move rigidly with a maximum displacement of $0.5 d_{0}$. Moreover, the cluster is allowed to rotate (with a maximum angular change of $10^{\circ}$ per step) around its center of mass. The roto-translation is expected to favor more compact aggregates with respect to a simple translation. After reaching equilibrium (normally about 300000 iterations are required), the system is switched to a conventional algorithm to evaluate the mean values of the quantities of interest (as the magnetization). For a discussion on the balance of the cluster moving algorithm we remand the reader to the work of A. Satoh. ${ }^{13}$

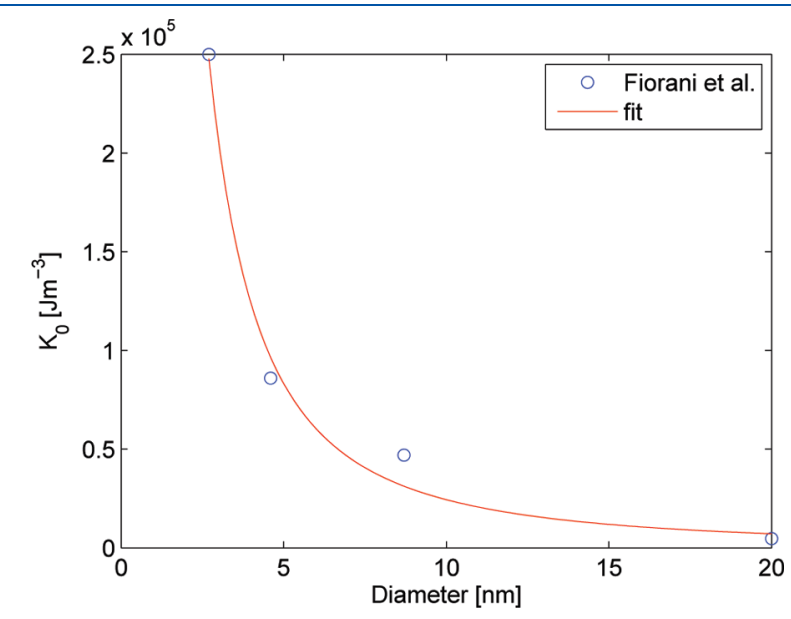

Figure 4. Dependence of the effective anisotropy on the particle diameter (after Fiorani et al. ${ }^{17}$ ). The fit was done using a power law function.

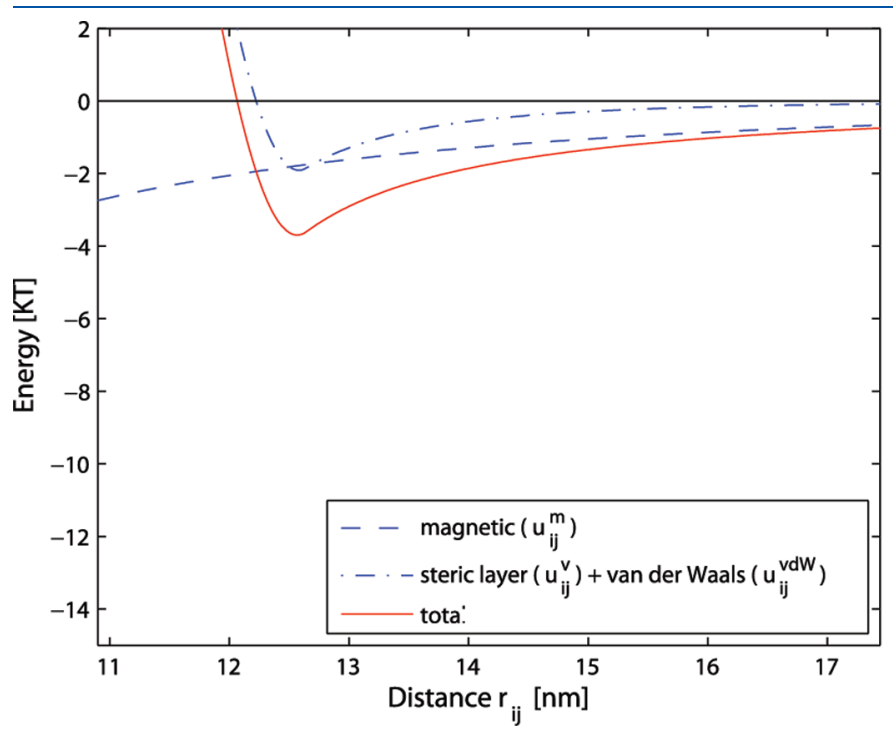

A critical parameter is the thickness of the surfactant layer $\delta$. Increasing the thickness of the capping layer makes the chain formation progressively unfavorable. ${ }^{18}$ Preliminary simulations on $15 \mathrm{~nm}$ particles indicated that a volume concentration greater than 0.005 is needed in order to start seeing agglomerated structures. ${ }^{19}$ In the following, the simulations are carried out at a 0.1 concentration. In our simulation, the polymer acts as a model fluid with some characteristics:

- During film deposition (Figure 6), the polymer is considered as a fluid with no viscosity. No dissipation is produced when the particles move or rotate. Nevertheless, the polymer can have an electrostatic polarizability, that can reduce the electrostatic interaction between particles. This effect can be taken into account by reducing the Hamaker's constant $A$ in eq $8 .{ }^{20}$ However, the polymer has negligible magnetic response. Moreover, the chemical interaction of the polymer with the particle surfactant can alter the thickness $\delta$ of the capping layer, and consequently the effective distances between particles.

- When the film has dried (Figure 7), the polymer acts as a solid medium. The particles cannot move or rotate anymore. Only the magnetic moments of the particles are free to turn.

\section{RESULTS AND DISCUSSION}

Chains Formation. The resulted structures, with and without the application of the magnetic field, are shown in Figure 6 for the $17 \mathrm{~nm}$ particles (top panels), and for the $11 \mathrm{~nm}$ particles (bottom panels). The parameters used in the simulations are listed in Table 1. The number of iterations in the simulation was 500000 , with cluster movements performed every 100 iterations. A final run with 100000 iterations with only single particles movements was carried out to calculate average values (magnetic moments and anisotropy axes). It is clear that aggregation in nanochains is favored for larger particles (higher magnetic moments), but it is critical for small particles. Because of the weak magnetic interparticle dipolar interactions, the simulation predicts elongated structures in the direction of the field (vertical direction in the figures), for the $11 \mathrm{~nm}$ particles, with a much more clear chains formation for the $17 \mathrm{~nm}$ particles This is due to the fact that for

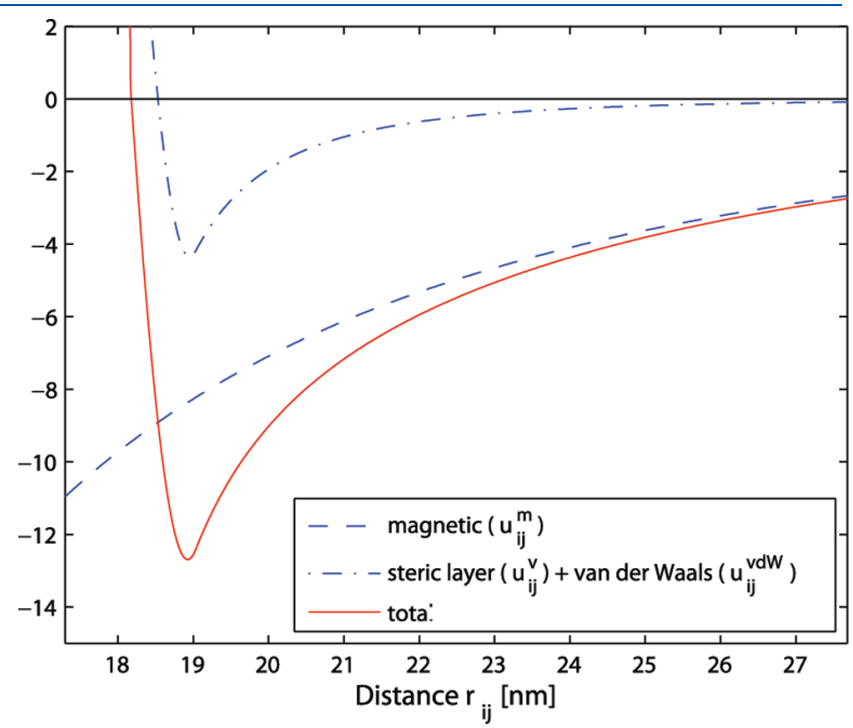

Figure 5. Interparticle potentials for two particles with diameter $d_{0}=11 \mathrm{~nm}$ (left) and $d_{0}=17 \mathrm{~nm}$ (right) (Table 1 for details). 

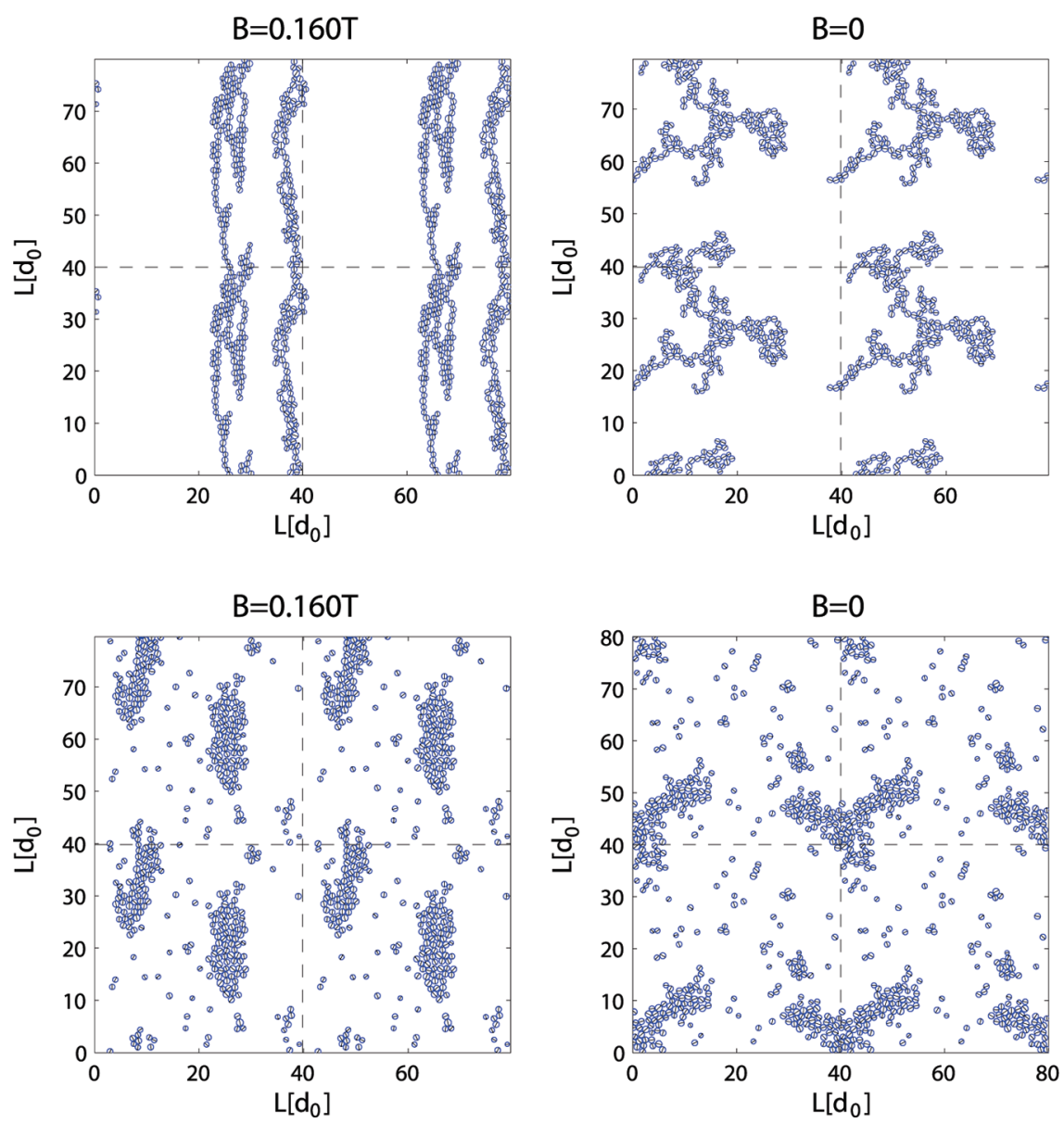

Figure 6. (top) Comparison of the simulations for a $d_{0}=17 \mathrm{~nm}$ particles dispersion at concentration 0.1 under a vertical magnetic field $B=0.160 \mathrm{~T}($ left $)$, and with no field (right); (bottom) comparison of the simulations for a $d_{0}=11 \mathrm{~nm}$ particles dispersion at concentration 0.1 under a vertical magnetic field $B=0.160 \mathrm{~T}($ left $)$, and with no field (right).
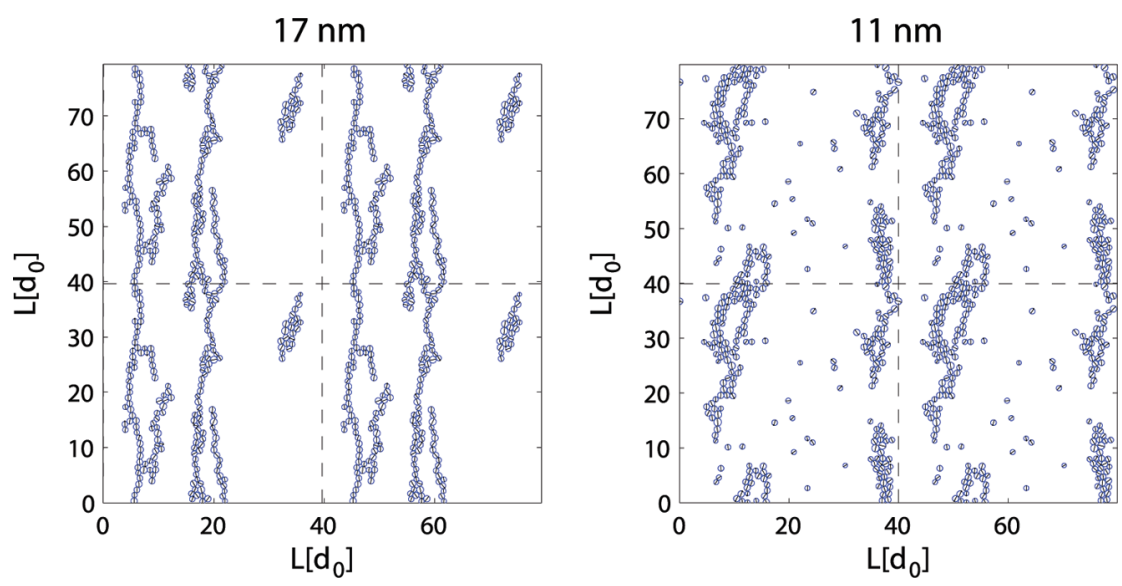

Figure 7. Comparison of the simulations for $d_{0}=17 \mathrm{~nm}($ left $)$, and $d_{0}=11 \mathrm{~nm}($ right $)$ particles at concentration 0.1 under a vertical magnetic field $B=$ $0.160 \mathrm{~T}$, considering lower values for $\delta$ and $A$ due to the presence of the polymer.

small particles (Figure 5), the magnetic and van der Waals terms are comparable, whereas for the bigger particle the magnetic term prevails. With no magnetic field, cluster with no elongated shape are obtained as expected. Surprisingly, the experiments have shown chains formation also for the small particles. ${ }^{56}$ This result can be partly explained by the presence of the polymer. First, the polymer can reduce the effective electrostatic interaction between particles (reducing $A$ in eq 8). Second, the mixing of the polymer with the nanoparticles can result in a reduction of the capping layer thickness $\delta$, reducing the particle distance, and favoring aggregation into cluster, a fundamental step for the chain formation. ${ }^{18}$ The same analysis of the FFT of the TEM images 
Table 1. Parameters Values Used in the Simulations ${ }^{a}$

$\begin{array}{llll}d_{0} & 11 & 17 & \mathrm{~nm} \\ \delta & 0.08 d_{0} & 0.05 d_{0} & \\ & \left(0.03 d_{0}\right) & \left(0.02 d_{0}\right) & \\ \xi_{0} & 10 & 40 & \\ \lambda_{0} & 1.4 & 5.5 & \\ \lambda_{\mathrm{v}} & 375 & 940 & \mathrm{Jm}^{-3} \\ & (1800) & (4700) & \mathrm{J} \\ K_{0} & 2.4 \times 10^{4} & 1.2 \times 10^{4} & \\ A & 10^{-9} & 10^{-9} & \end{array}$

${ }^{a} \mathrm{~A}$ standard deviation in the diameters of $0.1 d_{0}$ was used. Between brackets the parameters used for simulating the presence of the polymer. The values for $K_{0}$ are derived from the fit of Figure 4.

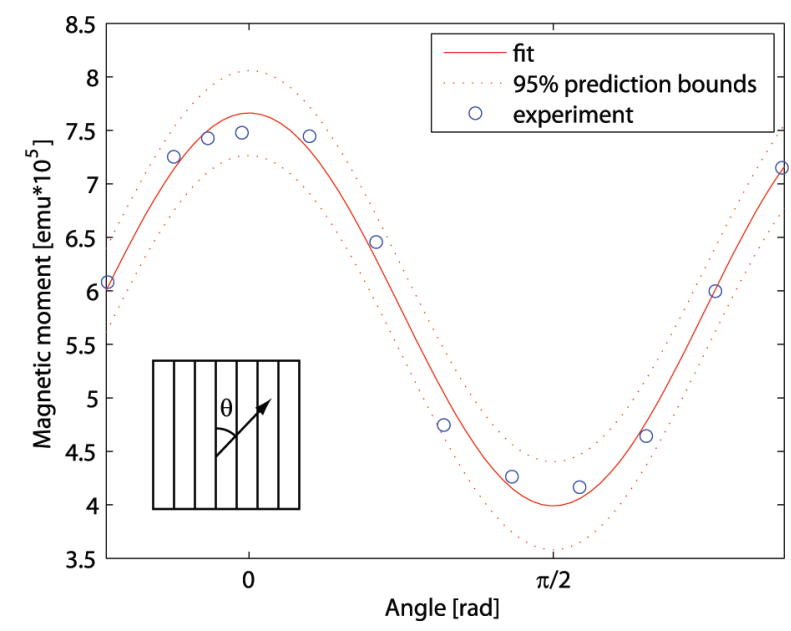

Figure 8. In plane magnetization of the nanocomposite film of Figure 1, measured at RT in a SQUID magnetometer. ${ }^{5}$

from films slices (as the one in Figure 1), revealed that the interparticle distance is reduced with respect to the dispersed particle in the pristine solution (about $1 \mathrm{~nm}$ lower). A second simulation with modified parameters (from Table 1) was run to verify these assumptions, and is presented in Figure 7. As expected, more elongated chains are formed also for the $11 \mathrm{~nm}$ particles, pointing to a not negligible effect of the interstitial polymer.

Magnetization. SQUID measurements revealed that the dried nanocomposite films show magnetic anisotropy even at $300 \mathrm{~K}^{5}$ Figure 8 shows the magnetization measured in the plane of the nanochains, obtained with a small field (0.005 T). A fit of the type described in 5 was performed, and a resulting anisotropy ratio in the magnetization of $1.9 \pm 0.3$ between parallel and perpendicular magnetization (with respect to the nanochains direction) derived. A simulation of the dc SQUID measurement using the same small field can be done using the Monte Carlo algorithm, starting from the configuration in Figure 7 , and applying the small field $(B=0.005 \mathrm{~T}$, resulting in $\xi_{0}=1.3$ for $d_{0}=17.3 \mathrm{~nm}$, and $\xi_{0}=0.33$ for $\left.d_{0}=10.9 \mathrm{~nm}\right)$ in the chosen directions (parallel and perpendicular to the chains). The anisotropy axes of the particles are now frozen (the polymer has dried), that is particles cannot rotate anymore, whereas magnetic moments can turn according to eq 1 . No cluster movements are allowed either, due to the dried polymer medium. To let the system reaching equilibrium, we performed 300000 iterations, and calculated the average values (magnetic moments) in the last 50000 iterations. The simulations gave a ratio between parallel and perpendicular magnetization of $2.1 \pm 0.4$, for the $17 \mathrm{~nm}$ particles, and $2.5 \pm 1.1$ for the $11 \mathrm{~nm}$ particles, in very good agreement with the measured value.

Despite the success of this simple model in describing some macroscopic behavior of the nanocomposite films, some points need further consideration:

- We have described a 3D films with a 2D model. This can be a rough approximation if the interaction between the nanochains is negligible, as found experimentally. ${ }^{5}$

- The embedded medium was simply considered as a fluid with no viscosity, whereas in practice its viscosity is increasing with time. Despite the nice results of the Monte Carlo simulations, a molecular dynamic approach, ${ }^{18}$ implementing the model for the forces, while being more time-consuming, could give interesting results, possibly predicting the length of the wires, or the thickness of the segregation layer of particles often found at the surface. ${ }^{6}$

- We have measured the interparticle distance in the first simulation (Figure 6) from drop casted solution on a TEM grid. If the adhesion force with the carbon film is not negligible, the distance between the particles should be correctly measured by imaging the frozen solution on a holey grid, and possibly from a reconstructed tomographic volume. In the measurement of the particle distances in the dried films sections, we restricted the FFT analysis on regions where only a single $2 \mathrm{D}$ layer of particles from a wire was visible.

The wires can be imaged using magnetic force microscopy $(\mathrm{MFM})^{6}$ in the so-called NAP mode, probably due to the longrange interaction between the tip and the wires. Simulations as the ones presented here can be used to calculate a virtual MFM image, and verify if the small magnetic interaction is sufficient to produce detectable contrast, as for the case of the wider studied patterned permalloy films, ${ }^{21}$ or if other terms are responsible for the long-range interaction.

\section{CONCLUSIONS}

Agglomerated structures of superparamagnetic nanoparticles under the influence of a magnetic field were simulated making use of a Monte Carlo 2D algorithm. We included an uniaxial effective anisotropy, and the van der Waals attraction that cannot be neglected for nanometer sized particles. Aggregation into chainlike structures is demonstrated in the simulations, in agreement with experimental evidence obtained on iron oxide nanoparticles in polymers films. The interstitial polymer can influence the electrostatic interaction between particles and the capping layer thickness, with an effect on chains formation more pronounced for smaller particles. The magnetization anisotropy of the final film can be semiquantitatively predicted using a simple models for the particles interactions.

\section{AUTHOR INFORMATION}

\section{Corresponding Author}

*E-mail: giovanni.bertoni@iit.it.

\section{ACKNOWLEDGMENT}

The authors thank the group leaded by Prof. Gatteschi at INSTM of the University of Florence for the SQUID measurements, and 
Dr. R. Buonsanti and Dr. P.D. Cozzoli of National Nanotechnology Laboratory (NNL), CNR-Istituto di Nanoscienze, Università del Salento, for providing the iron oxide particles.

\section{REFERENCES}

(1) Michler, G. Electron Microscopy of Polymers; Springer Laboratory; Springer-Verlag: Berlin Heidelberg, 2008.

(2) Millan, A.; Palacio, F.; Falqui, A.; Snoeck, E.; Serin, V.; Bhattacharjee, A.; Ksenofontov, V.; Gütlich, P.; Gilbert, I. Acta Mater. 2007, 55, 2201-2209.

(3) Gilbert, I.; Millán, A.; Palacio, F.; Falqui, A.; Snoeck, E.; Serin, V. Polyhedron 2003, 22, 2457-2461, Proceedings of the 8th International Conference on Molecule-Based Magnets (ICMM 2002).

(4) Ausanio, G.; Barone, A.; Hison, C.; Iannotti, V.; Luponio, C., Jr.; Lanotte, L. Sens. Actuators, A 2006, 129, 25-28EMSA 2004 - Selected Papers from the 5th European Magnetic Sesors and Actuators Conference - EMSA 2004, Cardiff, UK, 4-6 July 2004.

(5) Fragouli, D.; Buonsanti, R.; Bertoni, G.; Sangregorio, C.; Innocenti, C.; Falqui, A.; Gatteschi, D.; Cozzoli, P. D.; Athanassiou, A.; Cingolani, R. ACS Nano 2010, 4, 1873-1878, PMID: 20356062.

(6) Fragouli, D.; Torre, B.; Bertoni, G.; Buonsanti, R.; Cingolani, R.; Athanassiou, A. Microscopy Research and Technique 2010, 73, 952-958.

(7) Cozzoli, P. D.; Snoeck, E.; Garcia, M. A.; Giannini, C.; Guagliardi, A.; Cervellino, A.; Gozzo, F.; Hernando, A.; Achterhold, K.; Ciobanu, N.; Parak, F. G.; Cingolani, R.; Manna, L. Nano Lett. 2006, 6, 1966-1972.

(8) Néel, L. Ann. Geophys. 1949, 5, 99.

(9) Bean, C. P.; Livingston, J. D. J. Appl. Phys. 1959, 30, S120-S129.

(10) Rasband, W. Image, J. 1997-2009; http://rsb.info.nih.gov/ij/, Accessed February 18, 2010.

(11) Vlugt, T.; van der Eerden, J.; Dijkstra, M.; Smit, B.; Frenkel, D. Introduction to Molecular Simulation and Statistical Thermodynamics. 2008; http://www.phys.uu.nl/ vlugt/imsst, Accessed July 10, 2010.

(12) Metropolis, N.; Rosenbluth, A. W.; Rosenbluth, M. N.; Teller,

A. H.; Teller, E. J. Chem. Phys. 1953, 21, 1087-1092.

(13) Satoh, A. J. Colloid Interface Sci. 1992, 150, 461-472.

(14) Aoshima, M.; Satoh, A. J. Colloid Interface Sci. 2005, $288,475-488$.

(15) Tannous, C.; Gieraltowski, J. Eur. J. Phys. 2008, 29, 475.

(16) Morimoto, H.; Maekawa, T. J. Phys. A: Math. Gen. 2000, 33, 247.

(17) Fiorani, D.; Testa, A. M.; Lucari, F.; D’Orazio, F.; Romero, H. Phys. Rev. B: Condens. Matter 2002, 320, 122-126.

(18) Richardi, J.; Motte, L.; Pileni, M. P. Curr. Opin. Colloid Interface Sci. 2004, 9, 185-191.

(19) Bertoni, G.; Torre, B.; Fragouli, D.; Buonsanti, R.; Athanassiou, A.; Cingolani, R. Magnetically Assembled Nanowires Embedded in Polymers. 2010; IMC17, 17th IFSM Microscopy Congress, Rio de Janeiro, Brasil.

(20) Israelachvili, J. N. Intermolecular and Surface Forces, 2nd ed.; Academic Press: London, 1992; Chapter 2.1 pages 13-14.

(21) Gubbiotti, G.; Albini, L.; Carlotti, G.; Crescenzi, M. D.; Fabrizio, E. D.; Gerardino, A.; Donzelli, O.; Nizzoli, F.; Koo, H.; Gomez, R. D. J. Appl. Phys. 2000, 87, 5633-5635. 